\title{
Internal derangement of temporomandibular joint: role of arthocentesis with steriod
}

\author{
Altaf Hussain Malik \\ Department of Oral and Maxillofacial Surgery, Govt Dental College, University of Kashmir, Hazratbal, Srinagar 190006, JEK, India. \\ Address for correspondence: Dr. Altaf Hussain Malik, Department of Oral \& Maxillofacial Surgery, Govt Dental College, University of \\ Kashmir, Hazratbal, Srinagar 190006, J \& K, India. E-mail:drmalikaltaf@gmail.com
}

\begin{abstract}
Aim: The aim of this study was to compare the efficacy of arthrocentesis with arthrocentesis plus steroid in the treatment of temporomandibular joint (TMJ) internal derangements. Methods: Nine males and 11 females aged 17-39 years were enrolled in the study. The patients were complaining of limited mouth opening and TMJ pain. Arthrocentesis was performed under aseptic conditions. All patients were clinically evaluated before the procedure, and 1 week and 6 months after the procedure. Intensity of TMJ pain and maximal mouth opening was recorded at each follow-up visit. Results: Both groups showed significant improvement in mouth opening and a reduction in pain scores in the postoperative period; however, the addition of steroid did not improve the overall outcome of the procedure. Conclusion: Arthrocentesis is a simple and safe procedure for patients with internal derangement of the TMJ with closed lock. However, the outcome was not improved by the addition of steroid.
\end{abstract}

Key words:

Arthrocentesis, internal derangement, steroid, temporomandibular joint

\section{INTRODUCTION}

The temporomandibular joint (TMJ) is subject to many disorders commonly known as temporomandibular joint disorders (TMD). These disorders are accompanied by pain, limitation, and deviation in mandibular range of motion, TMJ sounds, headache, and facial pain. Among these, internal derangement and TMJ osteoarthritis are the most common disorders, ranging from normal mouth opening and clicking to varying degrees of pain, restricted mouth opening, and loss of functional activity. The term "internal derangement" was introduced by Hey in 1814 as a general orthopedic term for a localized mechanical fault in a joint, but was later used more specifically to describe

\begin{tabular}{|l|l|}
\hline \multicolumn{2}{|c|}{ Access this article online } \\
\hline Quick Response Code: & Website: \\
\hline & www.parjournal.net \\
\hline & \\
\hline
\end{tabular}

displacement of the TMJ disc. ${ }^{[1]}$ Arthrocentesis for the TMJ was introduced by Nitzan et al. in $1991^{[2]}$ and bridged the gap between surgical and nonsurgical treatment. ${ }^{[3]}$ It involved irrigation of the upper joint compartment with a therapeutic substance, releasing adhesions, and flushing out inflammatory substrates, thereby relieving pain and improving function. TMJ arthrocentesis signifies the lavage of the upper joint compartment with physiological saline or Hartmann's solution (Ringer's lactate) using a needle for in- and out-flow. ${ }^{[4]}$ Arthrocentesis can be performed either under low pressure using an elevated infusion bag or under normal pressure using a syringe. ${ }^{[1]}$ This technique was first introduced at the beginning of the 1990s and was derived directly from TMJ arthroscopy, based on the hypothesis that the most effective successful component of TMJ arthroscopy was merely that the patient was submitted to an intervention, and not based on all the complicated maneuvers intended to recapture the disc, fix the disc, and remove the adherences within the joint using tiny and sophisticated instruments. ${ }^{[2]}$ Arthrocentesis, as originally proposed, employed a technique involving the use of two needles that were inserted into the superior joint space at certain 
points; these points were termed "McCain's points" and were marked on a line drawn from the middle of the tragus to the lateral canthus. The entry points were marked along this canthotragal line. The first point, corresponding to the glenoid fossa, was marked $10 \mathrm{~mm}$ from the mid-tragus and $2 \mathrm{~mm}$ below the line, and the second point, corresponding to articular eminence, was marked $10 \mathrm{~mm}$ from the first point and $10 \mathrm{~mm}$ below the line. The simple flushing action in the joint may eliminate or reduce the effect of biochemical factors that contribute to inflammation and pain. Intra-articular corticosteroid injections are occasionally administered to alleviate inflammation. Intra-articular corticosteroid injection has an unpredictable prognosis and can also lead to local side-effects on the joint.

\section{METHODS}

A total of 20 patients (11 females and 9 males) aged $17-39$ years were enrolled in this study. The review board of University of Kashmir approved this study. The procedures followed were in accordance with the ethical standards of the responsible committee on human experimentation and with the Helsinki declaration. A detailed clinical examination was performed, along with any necessary investigations. All the patients selected for the study had been diagnosed with TMJ internal derangement with closed lock through clinical and radiographic examination (magnetic resonance imaging). Only such patients were taken for study in which all the conservative measures had failed. A written and verbal consent was obtained from the patients for treatment and associated complications, after the treatment outcome were fully explained to them. A visual analog scale (VAS) was used to score pain (range 1-10), where 1 denoted no pain at all, and 10 denoted very severe pain. These values were recorded at 1-week preoperatively, and at 6 months postoperatively. The maximal mouth opening (MMO) was evaluated and recorded (in $\mathrm{mm}$ ) pre- and post-operatively. The patients were divided into two groups, with 10 patients per group. One group underwent only arthrocentesis while the other group underwent arthrocentesis followed by a single injection of triamcinolone acetonide $(20 \mathrm{mg})$ into the joint. The results were compared both pre- and post-operatively. The entire procedure was performed by one surgeon and conducted under local anesthesia [Figure 1]. About $100 \mathrm{ml}$ of Ringer's lactate was used for arthrocentesis. After the lavage was completed, the needles were removed, and the patient's jaw was gently manipulated by the clinician in the vertical, protrusive and lateral excursions to help further release the disc and break the adhesions. The patients were then followed up at 1 week and 6 months. The results were then analyzed using an SPSS Statistics software package manufactured by IBM Corporation (Armonk, New York, U.S.). Postoperative changes in pain, lateral movements, and range of mouth opening were compared with the preoperative values using the Mann-Whitney U-test.

\section{RESULTS}

Before treatment, the mean VAS score was 6.6 and the mean MMO was $23 \mathrm{~mm}$. Posttreatment, the mean VAS pain score decreased to a mean value of 2.7 at 1 week and then to 1.2 at 6 months in the arthrocentesis-only group [Table 1], whereas the MMO increased to a mean value of $34 \mathrm{~mm}$ at 1 week and $42.1 \mathrm{~mm}$ at 6 months in the steroid group [Table 1], which indicates significant improvement in patient symptoms and complaints. However, the mean MMO after steroid injection increased from 23.9 to $42.2 \mathrm{~mm}$ [Table 1], and the mean pain score decreased from 6.7 to 1.11 [Table 2], though the difference between the two groups was not significant.

\section{DISCUSSION}

Temporomandibular disorders comprise a wide variety of disorders of the TMJ, masticatory muscles, or both, ${ }^{[5,6]}$ with the main symptoms presenting as pain and dysfunction. Pain associated with TMJ disorders may be due to vasoconstriction and release of nitric oxide, reactive oxygen species (ROS), and thiobarbituric acid. Elevated ROS levels in synovial fluid may result from mechanical stress and high pressures directed to the upper compartment during clenching and jaw movement. . $^{[7]}$ Lavage of the upper compartment by TMJ arthrocentesis forces apart the flexible disc from the fossa, washes away degraded particles containing inflammatory components and decreases intra-articular pressure. Furthermore, the elimination of nitric oxide and ROS relieves the pain.

The concept of TMJ arthrocentesis and lavage was first borne out of the successful use of TMJ arthroscopy

Table 1: Comparison of MMO between the two groups

\begin{tabular}{|c|c|c|c|c|}
\hline \multirow[t]{2}{*}{ Group } & \multirow{2}{*}{$\begin{array}{c}\text { Mean } \\
\text { preoperative } \\
\text { MMO (mm) }\end{array}$} & \multicolumn{2}{|c|}{$\begin{array}{c}\text { Mean postoperative } \\
\text { MMO }(\mathrm{mm})\end{array}$} & \multirow[t]{2}{*}{$P$ value } \\
\hline & & At 1-week & At 6 months & \\
\hline $\begin{array}{l}\text { Arthrocentesis } \\
\text { only }(n=10)\end{array}$ & 23 & 34 & 42.1 & 1.000 \\
\hline $\begin{array}{l}\text { Arthrocentesis } \\
\text { plus steroid }(n=10)\end{array}$ & 23.9 & 33.3 & 42.2 & \\
\hline
\end{tabular}

MMO: Maximum mouth opening; $n$ : Number of patients

Table 2: Comparison of pain between the two groups using a VAS

\begin{tabular}{|c|c|c|c|c|}
\hline \multirow[t]{2}{*}{ Group } & \multirow{2}{*}{$\begin{array}{c}\text { Mean } \\
\text { preoperative } \\
\text { pain }\end{array}$} & \multicolumn{2}{|c|}{$\begin{array}{c}\text { Mean postoperative } \\
\text { pain }\end{array}$} & \multirow[t]{2}{*}{$P$ value } \\
\hline & & At 1 -wee & 6 months & \\
\hline $\begin{array}{l}\text { Arthrocentesis } \\
\text { only }(n=10)\end{array}$ & 6.6 & 2.7 & 1.2 & 1.000 \\
\hline $\begin{array}{l}\text { Arthrocentesis } \\
\text { plus steroid }(n=10)\end{array}$ & 6.7 & 2.4 & 1.11 & \\
\hline
\end{tabular}




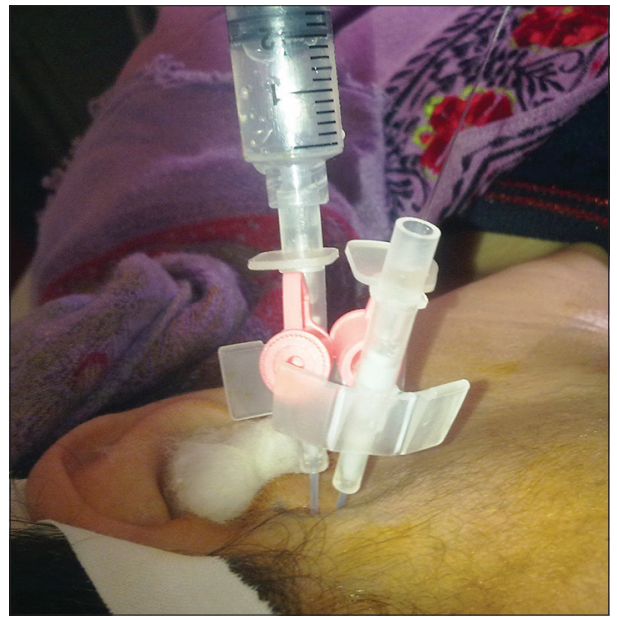

Figure 1: Arthrocentesis in progress

not only as a diagnostic tool, but also as a therapeutic technique, resulting in remarkable improvement in pain, jaw opening and function in selected patients simply by lavage of the superior joint space. ${ }^{[4]}$ The hydraulic distension provoked by the lavage pressure under the upper joint compartment with a large volume of saline has been considered the reason for the positive clinical outcomes in patients with sudden-onset closed lock. ${ }^{[8]} \mathrm{A}$ single-session arthrocentesis procedure was then proven effective also in improving pain and dysfunction in subjects affected by TMJ osteoarthritis, likely due to a thorough removal of catabolytes from the joint space. ${ }^{[6]}$ In the case of the closed lock, the central portion of healthy disc indeed separates from the fossa, leaving rims fastened to the surface of the eminence, which leads to increased negative pressure in the closed space between the fossa and disc. This pressure difference constitutes a force sufficient to keep the disc compressed against the fossa (the "suction cup effect"). ${ }^{[9]}$

In our study, the maximum mouth opening increased from 23 to $42.1 \mathrm{~mm}$ in the arthrocentesis-only group, whereas it increased from 23.9 to $42.2 \mathrm{~mm}$ in the steroid group [Table 1]. The VAS score decreased from 6.6 to 1.2 in the arthrocentesis group, whereas it decreased from 6.7 to 1.11 in the steroid group [Table 2]. The results did not support the clear superiority of one treatment protocol over the others to achieve pain management in TMJ inflammatory-degenerative joint disease over a short-term, namely a 6-month follow-up period. Findings suggested that neither statistically nor clinically significant differences existed between the treatment groups. This concurs with the study conducted by Manfredini who compared six different treatment protocols. All protocols were associated with positive outcomes, in line with the TMD literature highlighting improvements, at least to some extent. ${ }^{[10]}$ Murakami et al. compared arthrocentesis, arthroscopic surgery, and nonsurgical treatments in TMJ closed lock and found similar values for pain level and jaw dysfunction. ${ }^{[1]}$ They concluded that arthrocentesis, rather than being an alternative to arthroscopic surgery, would be indicated for patients with acute TMJ closed lock refractory to medication and mandibular manipulation. In their review, Al-Belasy and Dolwick have reported that no medication was used for intra-articular injection in 4 studies, steroid was used in 14 studies, and hyaluronic acid was used in 2 studies. $^{[9]}$

Complications are rare in arthrocentesis and occur more often with arthroscopy. ${ }^{[12]}$ Nevertheless, potential complications may develop with arthrocentesis, such as damage to capsular tissues and discal tissue, increased risk of the facial nerve injury, preauricular hematoma, middle ear injury, and intra-articular instrument breakage. Redundant injury of the capsule by needles can also aggravate inflammation in the joint and increase the incidence of solution extravasation to neighboring tissues when the arthrocentesis is finally performed. ${ }^{[13-16]}$ A rare case of extradural hematoma has also been reported with the conventional technique of arthrocentesis, which could have occurred because of blind triangulation of the needle. Arthrocentesis and arthroscopy are the primary treatments for patients who fail conservative methods of management of TMJ pain, restriction, and locking. An improvement in mouth opening is observed irrespective of the Wilkes score. There is a clear improvement in pain score based on this intervention, and as such, this management should be offered routinely. ${ }^{[17]}$ Our results concur with the study conducted by Xu et al., who showed that lavage and arthrocentesis helped to improve range of mouth opening and lateral movements, and reduce patient complaints. ${ }^{[18]}$ In the absence of a clear history of trauma, arthrocentesis should be the first-line treatment in patients aged under 25 years. ${ }^{[15]}$

The results of this study concur with those of other studies, which show that arthrocentesis improves range of mouth opening and relieves pain, but the addition of steroids does not help to alleviate the symptoms of TMJ derangements.

\section{CONCLUSION}

Temporomandibular joint arthrocentesis and lavage with manipulation is a simple, less invasive and less expensive technique than TMJ arthroscopy with low morbidity rates. It should be considered as an effective and efficient alternative to more invasive surgical procedures for a selected group of patients and as a minimally invasive, highly effective procedure in the treatment of patients with internal derangement of the TMJ with closed lock.

\section{REFERENCES}

I. Adams JC, Hamblen DL. Outline of Orthopedics. 13th ed. London, UK: Churchill Livingstone; 200 I. 
2. Nitzan DW, Dolwick MF, Martinez GA. Temporomandibular joint arthrocentesis: a simplified treatment for severe, limited mouth opening. J Oral Maxillofac Surg 1991;49: I 163-7.

3. Singh S, Shivamurthy DM, Varghese D. Re: Rahal A, et al.: single-puncture arthrocentesis-introducing a new technique and a novel device. J Oral Maxillofac Surg 2011;69:311.

4. Sanders B.Arthroscopic surgery of the temporomandibular joint: treatment of internal derangement with persistent closed lock. Oral Surg Oral Med Oral Pathol 1986;62:361-72.

5. Greene CS. The etiology of temporomandibular disorders: implications for treatment.J Orofac Pain 200I; I 5:93-105.

6. Carlsson GE. Epidemiology and treatment need for temporomandibular disorders.J Orofac Pain 1999;13:232-7.

7. Alpaslan C, Bilgihan A, Alpaslan GH, Güner B, Ozgür Yis M, Erbas D. Effect of arthrocentesis and sodium hyaluronate injection on nitrite, nitrate, and thiobarbituric acid-reactive substance levels in the synovial fluid. Oral Surg Oral Med Oral Pathol Oral Radiol Endod 2000;89:686-90.

8. Nitzan DW, Samson B, Better H. Long-term outcome of arthrocentesis for sudden-onset, persistent, severe closed lock of the temporomandibular joint. J Oral Maxillofac Surg 1997;55:15I-7.

9. Al-Belasy FA, Dolwick MF. Arthrocentesis for the treatment of temporomandibular joint closed lock: a review article. Int J Oral Maxillofac Surg 2007;36:773-82

10. Manfredini D, editor. Current Concepts on Temporomandibular Disorders. Berlin: Quintessence Publishing; 2010. p. 305-18.

I I. Murakami KI, lizuka T, Matsuki M,OnoT. Recapturing the persistent anteriorly displaced disk by mandibular manipulation after pumping and hydraulic pressure to the upper joint cavity of the temporomandibular joint. Cranio 1987;5: 17-24.

12. Frost DE, Kendell BD. Part II: the use of arthrocentesis for treatment of temporomandibular joint disorders.J Oral Maxillofac Surg 1999;57:583-7.

13. Carls FR, Engelke W, Locher MC, Sailer HF. Complications following arthroscopy of the temporomandibular joint: analysis covering a 10-year period (45I arthroscopies). J Craniomaxillofac Surg 1996;24:12-5.

14. McCain JP, Sanders B, Koslin MG, Quinn JH, Peters PB, Indresano AT Temporomandibular joint arthroscopy: a 6-year multicenter retrospective study of 4,83I joints. J Oral Maxillofac Surg 1992;50:926-30.

15. McCain JP. Complications of TMJ arthroscopy. J Oral Maxillofac Surg 1988;46:256.

16. McCain JP, de la Rua H. Foreign body retrieval: a complication of TMJ arthroscopy. Report of a case. J Oral Maxillofac Surg 1989;47: I 22 I-5.

17. Sidebottom AJ. Current thinking in temporomandibular joint management. BrJ Oral Maxillofac Surg 2009;47:91-4.

18. Xu Y, Lin H, Zhu P, Zhou W, Han Y, Zheng Y, Zhang Z. A comparative study between use of arthroscopic lavage and arthrocentesis of temporomandibular joint based on computational fluid dynamics analysis. PLoS One 20I 3;8:e78953.

How to cite this article: Malik AH. Internal derangement of temporomandibular joint: role of arthocentesis with steriod. Plast Aesthet Res 2014;1:29-32.

Source of Support: Nil, Conflict of Interest: None declared.

Received: 15-02-2014; Accepted: 29-05-2014 